\title{
On a discrete-time risk model with claim correlated premiums
}

\author{
Xueyuan $\mathrm{Wu}^{a *}$, Mi Chen ${ }^{b}$, Junyi $\mathrm{Guo}^{c}$ and Can $\operatorname{Jin}^{a}$ \\ ${ }^{a}$ Department of Economics, The University of Melbourne, VIC 3010, Australia \\ ${ }^{b}$ School of Mathematics and Computer Science, Fujian Normal University, Fuzhou 350007, China \\ ${ }^{c}$ School of Mathematical Sciences, Nankai University, Tianjin 300071, China
}

\begin{abstract}
This paper proposes a discrete-time risk model that has a certain type of correlation between premiums and claim amounts. It is motivated by the well-known bonus-malus system (also known as the No Claims Discount) in the car insurance industry. Such a system penalises policyholders at fault in accidents by surcharges, and rewards claim-free years by discounts. For simplicity, only up to three levels of premium are considered in this paper and recursive formulae are derived to calculate the ultimate ruin probabilities. Explicit expressions of ruin probabilities are obtained in a special case. The impact of the proposed correlation between premiums and claims on ruin probabilities is examined through numerical examples. In the end, the joint probability of ruin and deficit at ruin is also considered.
\end{abstract}

Keywords: Discrete-time risk model; No claims discount; Bonus-malus; Ruin probability; Deficit at ruin; Recursion

\section{Introduction}

The No Claims Discount (NCD) (or Bonus-Malus System) is a well-established system in general insurance industry worldwide, in particular for car insurances. For instance, in Australia, car insurance policyholders have the chance to renew

${ }^{*}$ Corresponding author. Email: xueyuanw@unimelb.edu.au; Tel.: 61-3-83447939 
their policies by paying discounted premiums if they made no claim at-fault in previous policy year. In another word, safe drivers are awarded by paying less premiums and "bad" drivers are penalised by paying more. In practice, the rules are fairly complicated and vary among insurers, but in general this arrangement encourages drivers to drive more safely and also leads to a reduction of the number of small claims. The Bonus-Malus has been studied extensively by many researchers in the past, see [2] - [8] and the references therein.

Motivated by the type of correlation between premiums and claims embedded in the NCD system, we consider a portfolio of insurance policies of which premiums are dependent on the claim history. Dufresne [3] considered the stationary distributions of a bonus-malus system and showed that it can be computed recursively. It is further shown that there is an intrinsic relationship between such a stationary distribution and the probability of ruin in the risk theoretical model. Moreover, continuous-time risk models with varying premiums, experience-based premiums or credibility updated premiums, have been studied in recent literature, see Afonso et al [1], Li et al [7] and references therein.

Before we introduce our risk model, we shall make some important assumptions about the mechanism of the NCD system for the given insurance portfolio. In general the NCD system works for individual policies, and each policyholder is assessed based on his/her own claim history. Although a risk model built on this ground can be studied using simulations, on the portfolio level the number of possible annual premium income levels would be far too many to consider by other means. Therefore, we adopt a commonly used approach in practice that is to set several bands which represent the key scenarios of total annual claims of the portfolio. Each band covers a range of possible total annual claims and a level of premium discount is assigned to it. The total premiums that is receivable next year can then be determined given the previous year's total claims band. Furthermore, for each band, we shall only adopt its best estimate of total claim amount rather than retaining the "band" purely for the sake of tractability of the risk model built upon this basis.

Let $\mathbb{N}^{+}=\{1,2, \ldots\}$. Denote $U_{n}$ the amount of surplus of the insurer at time $n, n \in \mathbb{N}$, which has the form

$$
U_{n}=u+\sum_{i=1}^{n} C_{i}-\sum_{i=1}^{n} X_{i},
$$

where $u \in \mathbb{N}$ is a constant initial surplus and $\left\{X_{i}\right\}_{i \in \mathbb{N}^{+}}$form an independent and identically distributed (i.i.d.) random variable sequence with $X_{i}$ denoting the total claim amount in period $i \in \mathbb{N}^{+}$. Under the above assumptions, in the present paper we shall assume that $X_{i}$ only takes values $0, M$ or $N, M<N \in \mathbb{N}^{+}$ 
with probabilities $q=1-p_{1}-p_{2}$ and $p_{1}, p_{2}\left(0<p_{1}, p_{2}<1\right)$ respectively. Here $N$ represents the high claims band and $M$ represents the low to medium claims band. And $C_{i}$ is the amount of premiums the insurer receives at the beginning of period $i$ satisfying the following conditions, for $n \in \mathbb{N}^{+}$:

$$
\begin{aligned}
& \operatorname{Pr}\left(C_{n+1}=\theta K_{1} \mid X_{n}=0\right)=1, \\
& \operatorname{Pr}\left(C_{n+1}=\Theta K_{1} \mid X_{n}=M\right)=1, \\
& \operatorname{Pr}\left(C_{n+1}=K_{1} \mid X_{n}=N\right)=1,
\end{aligned}
$$

where $\theta<\Theta$ are constants in $(0,1]$ with $1-\theta$ and $1-\Theta$ representing the percentages of premium discount and $K_{1}$ is a positive integer denoting the full premium level. So $\theta K_{1}$ and $\Theta K_{1}$ are just the discounted premium levels. For the proposed portfolio of insurance policies, these conditions imply that if in period $n$ there were claim amounts totaling either zero or $M$, then the insurance company would charge lower premiums to its policyholders in next time period. Otherwise, the policyholders need to pay full premiums at renewal. As mentioned before, this is just a simplified version and more realistic model setting could be considered in the future.

For a new insurance portfolio, it makes sense to charge full premiums for the first time period, i.e. $C_{1}=K_{1}$, so we define

$$
T=\min \left\{n \in \mathbb{N}^{+}: u+K_{1}+\sum_{i=2}^{n} C_{i}-\sum_{i=1}^{n} X_{i}<0\right\}
$$

to be the time of ruin with $\sum_{i=2}^{1}=0$. And $\psi(u)=\operatorname{Pr}(T<\infty)$ is the ultimate ruin probability for the discrete-time surplus process defined in (1). A trivial observation about the ruin probability is that $\psi(u)=1$, for any $u<0$. For computational purposes we shall further assume that $\Theta K_{1}=K_{2}$ and $\theta K_{1}=\kappa_{2}$ where $K_{2}$ and $\kappa_{2}$ are also positive integers. A positive safety loading condition for the model is

$$
\kappa_{2}+p_{1}\left(K_{2}-\kappa_{2}-M\right)+p_{2}\left(K_{1}-\kappa_{2}-N\right)>0,
$$

which is obtained by the given assumptions for premiums.

The rest of the paper is organised as follows. Section 2 considers the above defined ultimate ruin probability $\psi(u)$ and derives a recursion approach for computation purposes. Section 3 works on a simplified case where explicit results of $\psi(u)$ can be obtained. Discussions and numerical examples are provided in this section regarding the impact of the proposed correlation between premiums and claims on the ruin probabilities. And in section 4, the deficit at ruin, denoted by $\left|U_{T}\right|$, is considered and the techniques used are similar to previous sections. Some concluding remarks are given in the end in respect of the results obtained in this paper. 


\section{Some general results}

\subsection{Recursive formulae for $\psi(u)$}

In this section, we shall derive recursive formulae for the ruin probability $\psi(u)$ of the risk model (1). First of all, we need to introduce some supplementary ruin probabilities that are slightly modified versions of $\psi(u)$. From the above section we know that $\psi(u)$ is the probability of ruin when full premiums are receivable at the beginning. If the first period's total premium is at a discounted level, then the resulted probability of ruin will be higher. Since there are two levels of discounts assumed so far, we denote the probability of ruin with initial premiums $\kappa_{2}$ and $K_{2}$ by $\psi_{1}(u)$ and $\psi_{2}(u)$ respectively. Clearly, $\psi(u) \leq \psi_{2}(u) \leq \psi_{1}(u)$.

Considering all possible experience of model (1) in the first time period, we obtain the following recursion:

$$
\psi(u)=q \psi_{1}\left(u+K_{1}\right)+p_{1} \psi_{2}\left(u+K_{1}-M\right)+p_{2} \psi\left(u+K_{1}-N\right) .
$$

The right-hand side of (2) covers all three cases of claim occurrence in period 1. It is worth mentioning that if there is no claim in the first time period, then $U_{1}=u+K_{1}$, and $C_{2}=\kappa_{2}$. If we deduct an amount of $K_{1}-\kappa_{2}$ from $U_{1}$ and combine it with $C_{2}$ then it is equivalent to the surplus process being renewed at time 1 with initial surplus $u+\kappa_{2}$ and full premiums $K_{1}$. Therefore,

$$
\psi_{1}\left(u+K_{1}\right)=\psi\left(u+\kappa_{2}\right) .
$$

Similarly, one can see that

$$
\begin{aligned}
& \psi_{1}\left(u+K_{1}\right)=\psi_{2}\left(u+K_{1}+\kappa_{2}-K_{2}\right), \\
& \psi_{2}\left(u+K_{1}\right)=\psi\left(u+K_{2}\right) .
\end{aligned}
$$

Making use of the relationship (3) and examining the detailed ranges of $u$, we obtain the following result:

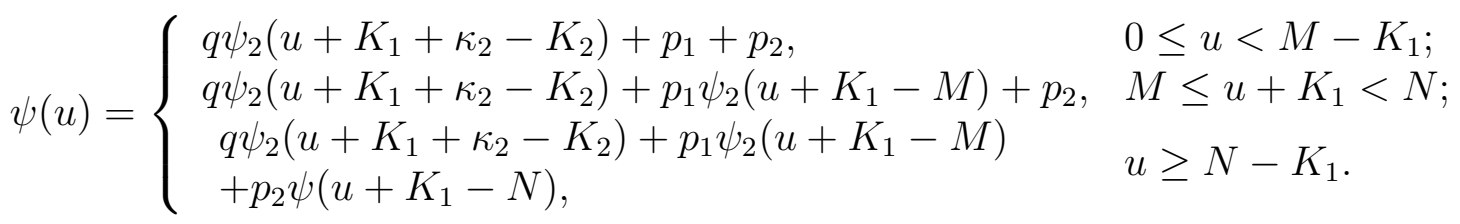

We can see from (5) that to determine $\psi(u)$ we need to know how to calculate 
$\psi_{2}(u)$ first. Following similar steps as above, we can get

$$
\begin{aligned}
\psi_{2}(u)= & q \psi_{1}\left(u+K_{2}\right)+p_{1} \psi_{2}\left(u+K_{2}-M\right)+p_{2} \psi\left(u+K_{2}-N\right) \\
= & \begin{cases}q \psi_{2}\left(u+\kappa_{2}\right)+p_{1}+p_{2}, & 0 \leq u<M-K_{2} \\
q \psi_{2}\left(u+\kappa_{2}\right)+p_{1} \psi_{2}\left(u+K_{2}-M\right)+p_{2}, & M \leq u+K_{2}<N \\
q \psi_{2}\left(u+\kappa_{2}\right)+p_{1} \psi_{2}\left(u+K_{2}-M\right) & u \geq N-K_{2} . \\
+p_{2} \psi\left(u+K_{2}-N\right), & u\end{cases}
\end{aligned}
$$

From the relationship (4) discussed previously, which implies that $\psi(u)=\psi_{2}(u+$ $\left.K_{1}-K_{2}\right), u \geq 0$, one can see that the last case in (6) can be rewritten as:

$$
\begin{aligned}
\psi_{2}(u)= & q \psi_{2}\left(u+\kappa_{2}\right)+p_{1} \psi_{2}\left(u+K_{2}-M\right) \\
& +p_{2} \psi_{2}\left(u+K_{1}-N\right), \quad u \geq N-K_{2} .
\end{aligned}
$$

Substituting it into (6) gives the following recursive formula in respect of $\psi_{2}(u)$ :

$$
\psi_{2}(u)= \begin{cases}\frac{1}{q}\left[\psi_{2}\left(u-\kappa_{2}\right)-p_{1}-p_{2}\right], & \kappa_{2} \leq u<M+\kappa_{2}-K_{2} ; \\ \frac{1}{q}\left[\psi_{2}\left(u-\kappa_{2}\right)-p_{1} \psi_{2}\left(u+K_{2}-\kappa_{2}-M\right)-p_{2}\right], & M \leq u-\kappa_{2}+K_{2}<N ; \\ \frac{1}{q}\left[\psi_{2}\left(u-\kappa_{2}\right)-p_{1} \psi_{2}\left(u+K_{2}-\kappa_{2}-M\right)\right. & u \geq N-K_{2}+\kappa_{2}, \\ \left.-p_{2} \psi_{2}\left(u+K_{1}-\kappa_{2}-N\right)\right], & \end{cases}
$$

where $\psi_{2}(i), i=0,1, \ldots, \kappa_{2}-1$ are initial values that need to be determined later on. Note that having calculated $\psi_{2}(u)$ we have also known the values of $\psi(u)$ by employing the relationship (4) again.

\subsection{Initial values}

In this subsection we shall determine the unknown initial values $\psi_{2}(i), i=0,1, \ldots$, $\kappa_{2}-1$, that were left over from the previous section. Wagner [9] considered a twostate Markov risk model where the state of a homogeneous Markov chain at any given time determines the corresponding claim amount. The method proposed in [9] to determine the initial values of ruin probabilities is of much use here.

Due to the number of initial values and the complexity embedded in the general setup of $N, M, K_{1}, K_{2}$, and $\kappa_{2}$, we ought to introduce some restrictions before we go on:

- $N-K_{1}$ and $M-K_{2}$ are both multiples of $\kappa_{2}$, i.e. $N-K_{1}=J_{1} \kappa_{2}$ and $M-K_{2}=J_{2} \kappa_{2}$ where $J_{i}, i=1,2$, are positive integers; 
- $K_{1}-K_{2}<\kappa_{2}$, since we normally expect the sum of the two discounted premium levels being higher than the full premium. Otherwise, either $\kappa_{2}$ is very small or both $K_{2}$ and $\kappa_{2}$ are less than half of $K_{1}$, none of which is reasonable in practice as the highest discount level in real world is generally around or moderately higher than $50 \%$. Let $I=K_{1}-K_{2}$, and as a result, we have $N-K_{2}=J_{1} \kappa_{2}+I$.

Then we have the following result.

Theorem 1 The initial values for the recursive formula (7) are

$$
\psi_{2}(i)= \begin{cases}\frac{p_{1} J_{2}+p_{2}\left(J_{1}+1\right)}{1-p_{1}}, & i=0,1, \ldots, I-1 ; \\ \frac{p_{1} J_{2}+p_{2} J_{1}}{q}, & i=I, I+1, \ldots, \kappa_{2}-1 .\end{cases}
$$

Proof. Let $p=p_{1}+p_{2}$. For $n \kappa_{2}>N-K_{2}+\kappa_{2}$ and $i=0,1, \ldots, I-1$, we have

$$
\begin{aligned}
& \psi_{2}\left(n \kappa_{2}+i\right)-\psi_{2}(i)=\sum_{j=1}^{n}\left[\psi_{2}\left(j \kappa_{2}+i\right)-\psi_{2}\left((j-1) \kappa_{2}+i\right)\right] \\
= & \sum_{j=1}^{J_{2}}\left[p \psi_{2}\left(j \kappa_{2}+i\right)-p\right]+\sum_{j=J_{2}+1}^{J_{1}+1}\left[p \psi_{2}\left(j \kappa_{2}+i\right)-p_{1} \psi_{2}\left(\left(j-J_{2}-1\right) \kappa_{2}+i\right)-p_{2}\right] \\
& +\sum_{j=J_{1}+2}^{n}\left[p \psi_{2}\left(j \kappa_{2}+i\right)-p_{1} \psi_{2}\left(\left(j-J_{2}-1\right) \kappa_{2}+i\right)-p_{2} \psi_{2}\left(\left(j-J_{1}-1\right) \kappa_{2}+i\right)\right] \\
= & p \sum_{j=1}^{n} \psi_{2}\left(j \kappa_{2}+i\right)-p_{1} \sum_{j=J_{2}+1}^{n} \psi_{2}\left(\left(j-J_{2}-1\right) \kappa_{2}+i\right) \\
& -p_{2} \sum_{j=J_{1}+2}^{n} \psi_{2}\left(\left(j-J_{1}-1\right) \kappa_{2}+i\right)-p J_{2}-p_{2}\left(J_{1}-J_{2}+1\right) \\
= & p \sum_{j=1}^{n} \psi_{2}\left(j \kappa_{2}+i\right)-p_{1} \sum_{j=0}^{n-J_{2}-1} \psi_{2}\left(j \kappa_{2}+i\right)-p_{2} \sum_{j=1}^{n-J_{1}-1} \psi_{2}\left(j \kappa_{2}+i\right)-p_{1} J_{2}-p_{2}\left(J_{1}+1\right) \\
= & p_{1} \sum_{j=n-J_{2}}^{n} \psi_{2}\left(j \kappa_{2}+i\right)-p_{1} \psi_{2}(i)+p_{2} \sum_{j=n-J_{1}}^{n} \psi_{2}\left(j \kappa_{2}+i\right)-p_{1} J_{2}-p_{2}\left(J_{1}+1\right) .
\end{aligned}
$$

When $u \rightarrow \infty, \psi_{2}(u)$ tends to zero, so letting $n \rightarrow \infty$ the above equation converges to

$$
-\psi_{2}(i)=-p_{1} \psi_{2}(i)-p_{1} J_{2}-p_{2}\left(J_{1}+1\right),
$$


which gives

$$
\psi_{2}(i)=\frac{p_{1} J_{2}+p_{2}\left(J_{1}+1\right)}{1-p_{1}}, i=0,1, \ldots, I-1 .
$$

It is less than 1 according to the safety loading condition given before.

Similarly, For $n \kappa_{2}>N-K_{2}+\kappa_{2}$ and $i=I, I+1, \ldots, \kappa_{2}-1$, we have

$$
\begin{aligned}
& \psi_{2}\left(n \kappa_{2}+i\right)-\psi_{2}(i)=\sum_{j=1}^{n}\left[\psi_{2}\left(j \kappa_{2}+i\right)-\psi_{2}\left((j-1) \kappa_{2}+i\right)\right] \\
= & \sum_{j=1}^{J_{2}}\left[p \psi_{2}\left(j \kappa_{2}+i\right)-p\right]+\sum_{j=J_{2}+1}^{J_{1}}\left[p \psi_{2}\left(j \kappa_{2}+i\right)-p_{1} \psi_{2}\left(\left(j-J_{2}-1\right) \kappa_{2}+i\right)-p_{2}\right] \\
& +\sum_{j=J_{1}+1}^{n}\left[p \psi_{2}\left(j \kappa_{2}+i\right)-p_{1} \psi_{2}\left(\left(j-J_{2}-1\right) \kappa_{2}+i\right)-p_{2} \psi_{2}\left(\left(j-J_{1}-1\right) \kappa_{2}+i\right)\right] \\
= & p \sum_{j=1}^{n} \psi_{2}\left(j \kappa_{2}+i\right)-p_{1} \sum_{j=J_{2}+1}^{n} \psi_{2}\left(\left(j-J_{2}-1\right) \kappa_{2}+i\right) \\
& -p_{2} \sum_{j=J_{1}+1}^{n} \psi_{2}\left(\left(j-J_{1}-1\right) \kappa_{2}+i\right)-p J_{2}-p_{2}\left(J_{1}-J_{2}\right) \\
= & p \sum_{j=1}^{n} \psi_{2}\left(j \kappa_{2}+i\right)-p_{1} \sum_{j=0}^{n-J_{2}-1} \psi_{2}\left(j \kappa_{2}+i\right)-p_{2} \sum_{j=0}^{n-J_{1}-1} \psi_{2}\left(j \kappa_{2}+i\right)-p_{1} J_{2}-p_{2} J_{1} \\
= & p_{1} \sum_{j=n-J_{2}}^{n} \psi_{2}\left(j \kappa_{2}+i\right)+p_{2} \sum_{j=n-J_{1}}^{n} \psi_{2}\left(j \kappa_{2}+i\right)-p \psi_{2}(i)-p_{1} J_{2}-p_{2} J_{1} .
\end{aligned}
$$

Letting $n \rightarrow \infty$ the above equation converges to

$$
-\psi_{2}(i)=-p \psi_{2}(i)-p_{1} J_{2}-p_{2} J_{1},
$$

which gives

$$
\psi_{2}(i)=\frac{p_{1} J_{2}+p_{2} J_{1}}{q}, i=I, I+1, \ldots, \kappa_{2}-1 .
$$

This completes the proof.

\subsection{A numerical example}

In this subsection a simple numerical example is given to illustrate the above method of calculating ruin probabilities recursively. 
Example 1. In this example, we consider a very simple insurance portfolio with uniform distributed aggregate claims in each year. We assume that the probability of zero claim in a year is $q=0.6$, and conditional on positive claims, the annual aggregate claims follows a $U(0,1000)$ distribution. We propose two claim bands, $(0,600]$ and $(600,1000]$. On the basis of this portfolio, we construct the following NCD system:

$$
\begin{aligned}
& N=800, \quad K_{1}=320, \quad p_{2}=0.16 \\
& M=K_{2}=300, \quad p_{1}=0.24 ; \\
& \kappa_{2}=160, \quad q=0.6 .
\end{aligned}
$$

The selection of above parameters has taken into account the restrictions introduced before in respect of $M, N, K_{1}, K_{2}$ and $\kappa_{2}$, which is mainly for the purpose of illustration. In real world, the determination of such parameters will be a far more complex practice than this example.

Using the recursive formula (7) together with the initial value results given in Theorem 1 , we calculate the values of ruin probability $\psi_{2}(u)$ for selected $u$ values. Accordingly, values of $\psi(u)$ are determined based on the relationship (4) between these two types of ruin probabilities. These results are summarised in Table 1.

\begin{tabular}{r|c|c||r|c|c}
\hline \multicolumn{3}{c}{$\psi_{2}(u)$} & $u=2100$ & 0.20051 & $\psi(2080)$ \\
\hline$u=0$ & 0.84211 & & 2260 & 0.17969 & $\psi(2240)$ \\
20 & 0.80000 & $\psi(0)$ & 2420 & 0.16104 & $\psi(2400)$ \\
180 & 0.74667 & $\psi(160)$ & 2580 & 0.14433 & $\psi(2560)$ \\
340 & 0.67911 & $\psi(320)$ & 2740 & 0.12935 & $\psi(2720)$ \\
500 & 0.59354 & $\psi(480)$ & 2900 & 0.11592 & $\psi(2880)$ \\
660 & 0.53848 & $\psi(640)$ & 3060 & 0.10389 & $\psi(3040)$ \\
820 & 0.48297 & $\psi(800)$ & 3220 & 0.09311 & $\psi(3200)$ \\
980 & 0.43067 & $\psi(960)$ & 3380 & 0.08345 & $\psi(3360)$ \\
1140 & 0.38723 & $\psi(1120)$ & 3540 & 0.07479 & $\psi(3520)$ \\
1300 & 0.34690 & $\psi(1280)$ & 3700 & 0.06703 & $\psi(3680)$ \\
1460 & 0.31061 & $\psi(1440)$ & 3860 & 0.06007 & $\psi(3840)$ \\
1620 & 0.27860 & $\psi(1600)$ & 4020 & 0.05384 & $\psi(4000)$ \\
1780 & 0.24963 & $\psi(1760)$ & 4180 & 0.04825 & $\psi(4160)$ \\
1940 & 0.22369 & $\psi(1920)$ & & \multicolumn{3}{|c}{} \\
\hline
\end{tabular}

Table 1: Some values of $\psi_{2}(u)$ and $\psi(u)$ of Example 1.

\section{Remarks.}


2.1. Table 1 confirms that $\psi(u)$ is actually a shifted version of $\psi_{2}(u)$ by $K_{1}-K_{2}$, i.e. $\psi(u)=\psi_{2}(u+20)$ in this example.

2.2. Each pair of adjacent $u$ values in the table give us an interval within which $\psi_{2}$ remains constant. For example, $\psi_{2}(u)=\psi_{2}(0)$ for $0 \leq u<20, \psi_{2}(u)=$ $\psi_{2}(20)$ for $20 \leq u<180$ and so on. Except the first one, all of the other intervals have the same length, i.e. $\kappa_{2}=160$.

2.3. The initial values of $\psi_{2}(u)$ are $\psi_{2}(u)=0.84211$ for $u=0, \ldots, 19$ and $\psi_{2}(u)=$ 0.8 for $u=20, \ldots, 159$.

2.4. To be able to determine the initial values, $\psi_{2}(u), u=0,1, \ldots, \kappa_{2}-1$, some restrictions were introduced at the beginning of previous subsection, i.e. $N-K_{1}$ and $M-K_{2}$ are both multiples of $\kappa_{2}$. Also we assumed that both $\theta K_{1}$ and $\Theta K_{1}$ are positive integers in Section 1. These assumptions have put restrictions on the values of $M, N, K_{1}, K_{2}$ and $\kappa_{2}$. When the given problem does not satisfy these assumptions, eg the discounted premiums are not integers, we need to work out some satisfactory values for $N, K_{1}$ and $K_{2}$ to get a close enough fit to the given real problem and then to convert the obtained probabilities to the original ruin problem. More detailed discussions will be given later on in a simplified case.

\section{A simplified case}

In this section we shall consider an insurance portfolio with a simplified NCD system, i.e. with only two levels of premiums instead of three. This case is built simply by allowing $p_{1}=0$ and $\kappa_{2}=K_{2}$ in the model studied in previous sections. As a result, the aggregate claims amount is either 0 or $N$ in each time period, which is less meaningful in real practice. However, by doing this, the original set of parameters reduces to a smaller set containing $N, K_{1}, K_{2}, q$, and $p$ (instead of $p_{2}$ ), which enables us to derive some explicit results and study the impact of discount in premiums on ruin probabilities more easily.

\subsection{Main results}

Firstly, we obtain the following recursion of ruin probability $\psi(u)$.

Theorem 2 The ultimate ruin probability, $\psi(u), u \geq K_{2}$, of risk model (1) satis- 
fies the following recursive formula:

$\psi(u)= \begin{cases}1-\frac{1}{q^{j u}}\left[1-\psi\left(i_{u}\right)\right], & K_{2} \leq u<N+K_{2}-K_{1}, \\ \frac{1}{q}\left[\psi\left(u-K_{2}\right)-p \psi\left(u+K_{1}-K_{2}-N\right)\right], & u \geq N+K_{2}-K_{1},\end{cases}$

where integers $i_{u}$ and $j_{u}$ are the remainder and quotient from dividing $u$ by $K_{2}$, i.e. $u=i_{u}+j_{u} K_{2}, 0 \leq i_{u} \leq K_{2}-1, j_{u} \geq 1$, with initial values $\psi\left(i_{u}\right), i_{u}=$ $0,1, \ldots, K_{2}-1$, yet to determine.

Proof. Considering all possible experience of model (1) in the first time period, we obtain the following recursion:

$$
\psi(u)=q \psi\left(u+K_{2}\right)+p \psi\left(u+K_{1}-N\right),
$$

which is a reduced version of result (2). One obvious difference here is that no supplementary probabilities of ruin are needed any more if we examine the no claim case in the same way as in Section 2.

Next we shall examine two ranges of $u$. For $0 \leq u<N-K_{1}$, equation (10) becomes

$$
\psi(u)-q \psi\left(u+K_{2}\right)=p .
$$

And for $u \geq N-K_{1}$, we have

$$
\psi(u)-q \psi\left(u+K_{2}\right)=p \psi\left(u+K_{1}-N\right) .
$$

Combining (11)-(12) gives a recursive formula for $\psi(u)$

$\psi(u)= \begin{cases}\frac{1}{q}\left[\psi\left(u-K_{2}\right)-p\right], & K_{2} \leq u<N+K_{2}-K_{1}, \\ \frac{1}{q}\left[\psi\left(u-K_{2}\right)-p \psi\left(u+K_{1}-K_{2}-N\right)\right], & u \geq N+K_{2}-K_{1},\end{cases}$

with initial values $\psi(u), u=0,1, \ldots, K_{2}-1$. Based on the first part of formula (9) one can obtain, for $K_{2} \leq u \leq K_{2}+N-K_{1}-1$,

$$
\psi(u)=\frac{\psi\left(i_{u}\right)+q^{j_{u}}-1}{q^{j_{u}}}=1-\frac{1}{q^{j_{u}}}\left[1-\psi\left(i_{u}\right)\right] .
$$

This completes the proof.

Having obtained the recursive formula for $\psi(u)$ in Theorem 2, next we need to determine the initial values $\psi\left(i_{u}\right), i_{u}=0,1, \ldots, K_{2}-1$. The derivations again follow [9]. Similar restrictions on the parameters exist, i.e. $N-K_{1}=J_{1} K_{2}$ where $J_{1}$ is a positive integer. Then we have the following result. 
Theorem 3 The initial values for the recursive formula (9) are

$$
\psi\left(i_{u}\right)=\frac{p J_{1}}{1-p}, \quad i_{u}=0,1, \ldots, K_{2}-1
$$

Proof. For $n K_{2}>K_{2}+N-K_{1}$, we have

$$
\begin{aligned}
& \psi\left(n K_{2}\right)-\psi(0)=\sum_{j=1}^{n}\left[\psi\left(j K_{2}\right)-\psi\left((j-1) K_{2}\right)\right] \\
= & \sum_{j=1}^{J_{1}}\left[p \psi\left(j K_{2}\right)-p\right]+\sum_{j=J_{1}+1}^{n}\left[p \psi\left(j K_{2}\right)-p \psi\left((j-1) K_{2}+K_{1}-N\right)\right] \\
= & p \sum_{j=1}^{n} \psi\left(j K_{2}\right)-p \sum_{j=0}^{n-J_{1}-1} \psi\left(j K_{2}\right)-p J_{1} \\
= & p \sum_{j=n-J_{1}}^{n} \psi\left(j K_{2}\right)-p \psi(0)-p J_{1} .
\end{aligned}
$$

When $u \rightarrow \infty, \psi(u)$ tends to zero, so letting $n \rightarrow \infty$ the above equation converges to

$$
-\psi(0)=-p \psi(0)-p J_{1}
$$

which gives $\psi(0)=\frac{p J_{1}}{1-p}$. It is less than 1 according to the safety loading condition.

Similarly, for $i_{u}=1,2, \ldots, K_{2}-1, J_{1} K_{2}+i_{u} \leq K_{2}+N-K_{1}-1$ also holds. Therefore,

$$
\begin{aligned}
& \psi\left(n K_{2}+i_{u}\right)-\psi\left(i_{u}\right)=\sum_{j=1}^{n}\left[\psi\left(j K_{2}+i_{u}\right)-\psi\left((j-1) K_{2}+i_{u}\right)\right] \\
= & \sum_{j=1}^{J_{1}}\left[p \psi\left(j K_{2}+i_{u}\right)-p\right]+\sum_{j=J_{1}+1}^{n}\left[p \psi\left(j K_{2}+i_{u}\right)-p \psi\left((j-1) K_{2}+i_{u}+K_{1}-N\right)\right] \\
= & p \sum_{j=n-J_{1}}^{n} \psi\left(j K_{2}+i_{u}\right)-p \psi\left(i_{u}\right)-p J_{1} .
\end{aligned}
$$

Letting $n \rightarrow \infty$ in the above equation and solving for $\psi\left(i_{u}\right)$ gives

$$
\psi\left(i_{u}\right)=\frac{p J_{1}}{1-p}
$$


which is equal to $\psi(0)$. This completes the proof.

Remark. Note that the result (14) cannot be obtained directly by simply letting $p_{1}=0$ in the result (8), as the ruin probabilities involved are different. However, one can verify that (14) is a special case of (8) making use of the relationship between $\psi$ and $\psi_{2}$ discussed previously.

Under the assumptions of Theorem $2 \& 3$, it is of great interest to also obtain an explicit expression of $\psi(u)$.

Theorem 4 The ruin probability $\psi(u), u \geq K_{2}$, of risk model (1) satisfies the following expression:

$$
\psi(u)=1-\frac{q-p J_{1}}{q^{j_{u}+1}}\left[1+\sum_{n=1}^{\beta}\left(-p q^{J_{1}}\right)^{n} \sum_{k=1}^{j_{u}-n J_{1}-n+1} a_{n}(k)\right]
$$

where $\beta$ is the quotient from dividing $j_{u}$ by $J_{1}+1$ and $a_{n}(k) \hat{=} \sum_{j=1}^{k} a_{n-1}(j), k=$ $1, \ldots, j_{u}-n J_{1}-n+1$ with $a_{1}(i)=1, i=1, \ldots, j_{u}-J_{1}$.

Proof. To prove this result we divide the possible $u$ values into the following ranges in terms of $\beta$ values and consider them one by one.

$$
\begin{aligned}
\beta & =0: 1 \leq j_{u}<J_{1}+1 \Rightarrow K_{2} \leq u<K_{2}+N-K_{1} \\
\beta & =1: J_{1}+1 \leq j_{u}<2\left(J_{1}+1\right) \Rightarrow K_{2}+N-K_{1} \leq u<2\left(K_{2}+N-K_{1}\right) \\
& \vdots \\
\beta & =m: m\left(J_{1}+1\right) \leq j_{u}<(m+1)\left(J_{1}+1\right) \\
& \Rightarrow m\left(K_{2}+N-K_{1}\right) \leq u<(m+1)\left(K_{2}+N-K_{1}\right)
\end{aligned}
$$

First of all, for the convenience of derivation, we define a function $\xi_{i_{u}, j_{u}}$ which equates $\psi(u)$. Then under the assumption of Theorem 3, the result in Theorem 2 can be rewritten as

$$
\xi_{i_{u}, j_{u}}= \begin{cases}1-\frac{1}{q^{j_{u}}}\left[1-\psi\left(i_{u}\right)\right] & 1 \leq j_{u}<J_{1}+1, \\ \frac{1}{q} \xi_{i_{u}, j_{u}-1}-\frac{p}{q} \xi_{i_{u}, j_{u}-1-J_{1}} & j_{u} \geq J_{1}+1 .\end{cases}
$$

By Theorem 3, the first half of the right-hand side in the above formula shows that the result (15) holds for $\beta=0$.

When $\beta=1$, i.e. $J_{1}+1 \leq j_{u}<2\left(J_{1}+1\right)$, we have

$$
\begin{aligned}
\xi_{i_{u}, j_{u}} & =\frac{1}{q} \xi_{i_{u}, j_{u}-1}-\frac{p}{q} \xi_{i_{u}, j_{u}-1-J_{1}} \\
& =\frac{1}{q} \xi_{i_{u}, j_{u}-1}-\frac{p}{q}\left[1-\frac{1}{q^{j_{u}-1-J_{1}}}\left(1-\psi\left(i_{u}\right)\right)\right] .
\end{aligned}
$$


Let $\phi(u)=1-\psi(u)$, we go on with the above derivation and obtain

$$
\begin{aligned}
\xi_{i_{u}, j_{u}} & =\frac{1}{q} \xi_{i_{u}, j_{u}-1}-\frac{p}{q}+\frac{p}{q^{j_{u}-J_{1}}} \phi\left(i_{u}\right) \\
& =\frac{1}{q}\left[\frac{1}{q} \xi_{i_{u}, j_{u}-2}-\frac{p}{q}+\frac{p}{q^{j_{u}-1-J_{1}}} \phi\left(i_{u}\right)\right]-\frac{p}{q}+\frac{p}{q^{j_{u}-J_{1}}} \phi\left(i_{u}\right) \\
& =\frac{1}{q^{2}} \xi_{i_{u}, j_{u}-2}-\left(\frac{p}{q}+\frac{p}{q^{2}}\right)+\frac{2 p}{q^{j_{u}-J_{1}}} \phi\left(i_{u}\right) \\
& \vdots \\
& =\frac{1}{q^{j_{u}-J_{1}}} \xi_{i_{u}, J_{1}}-\sum_{k=1}^{j_{u}-J_{1}} \frac{p}{q^{k}}+\frac{\left(j_{u}-J_{1}\right) p}{q^{j_{u}-J_{1}}} \phi\left(i_{u}\right) \\
& =\frac{1}{q^{j_{u}-J_{1}}}\left[1-\frac{1}{q^{J_{1}}} \phi\left(i_{u}\right)\right]+\frac{\left(j_{u}-J_{1}\right) p}{q^{j_{u}-J_{1}}} \phi\left(i_{u}\right)+1-\frac{1}{q^{j_{u}-J_{1}}} \\
& =1-\frac{\phi\left(i_{u}\right)}{q^{j_{u}}}\left[1-\left(j_{u}-J_{1}\right) p q^{J_{1}}\right]
\end{aligned}
$$

which coincide with (15) given $\beta=1$. Now we assume that (15) holds for $\beta=m$, i.e. for $m\left(J_{1}+1\right) \leq j_{u}<(m+1)\left(J_{1}+1\right)$,

$$
\xi_{i_{u}, j_{u}}=1-\frac{q-p J_{1}}{q^{j_{u}+1}}\left[1+\sum_{n=1}^{m}\left(-p q^{J_{1}}\right)^{n} \sum_{k=1}^{j_{u}-n J_{1}-n+1} a_{n}(k)\right] .
$$

By induction, to complete our proof we only need to show that (15) also holds for $\beta=m+1$. Let $\alpha=j_{u}-\beta\left(J_{1}+1\right)$. Since

$$
\begin{aligned}
\xi_{i_{u}, j_{u}}= & \frac{1}{q} \xi_{i_{u}, j_{u}-1}-\frac{p}{q} \xi_{i_{u}, j_{u}-1-J_{1}} \\
= & \frac{1}{q} \xi_{i_{u}, j_{u}-1}-\frac{p}{q}\left\{1-\frac{q-p J_{1}}{q^{j_{u}-J_{1}}}\left[1+\sum_{n=1}^{m}\left(-p q^{J_{1}}\right)^{n j_{u}-(n+1) J_{1}-n} \sum_{k=1}^{m} a_{n}(k)\right]\right\} \\
= & \frac{1}{q}\left\{\frac{1}{q} \xi_{i_{u}, j_{u}-2}-\frac{p}{q}\left[1-\frac{q-p J_{1}}{q^{j_{u}-1-J_{1}}}\left(1+\sum_{n=1}^{m}\left(-p q^{J_{1}}\right)^{n} \sum_{k=1}^{j_{u}-1-(n+1) J_{1}-n} a_{n}(k)\right)\right]\right\} \\
& -\frac{p}{q}\left\{1-\frac{q-p J_{1}}{q^{j_{u}-J_{1}}}\left[1+\sum_{n=1}^{m}\left(-p q^{J_{1}}\right)^{n j_{u}-(n+1) J_{1}-n} \sum_{k=1}^{m} a_{n}(k)\right]\right\}
\end{aligned}
$$




$$
\begin{aligned}
& =\frac{1}{q^{2}} \xi_{i_{u}, j_{u}-2}+\frac{p\left(q-p J_{1}\right)}{q^{j_{u}+1-J_{1}}}\left[1+\sum_{n=1}^{m}\left(-p q^{J_{1}}\right)^{n} \sum_{k=1}^{j_{u}-1-(n+1) J_{1}-n} a_{n}(k)\right] \\
& +\frac{p\left(q-p J_{1}\right)}{q^{j_{u}+1-J_{1}}}\left[1+\sum_{n=1}^{m}\left(-p q^{J_{1}}\right)^{n} \sum_{k=1}^{j_{u}-(n+1) J_{1}-n} a_{n}(k)\right]-\left(\frac{p}{q}+\frac{p}{q^{2}}\right) \\
& =\frac{1}{q^{\alpha+1}} \xi_{i_{u}, m\left(J_{1}+1\right)+J_{1}}+\frac{(\alpha+1) p\left(q-p J_{1}\right)}{q^{j_{u}+1-J_{1}}}+\frac{p\left(q-p J_{1}\right)}{q^{j_{u}+1-J_{1}}} \sum_{n=1}^{m}\left(-p q^{J_{1}}\right)^{n} \\
& \times\left[\sum_{k=1}^{j_{u}-(n+1) J_{1}-n} a_{n}(k)+\sum_{k=1}^{j_{u}-1-(n+1) J_{1}-n} a_{n}(k)+\cdots+\sum_{k=1}^{j_{u}-\alpha-(n+1) J_{1}-n} a_{n}(k)\right] \\
& -\left(\frac{p}{q}+\frac{p}{q^{2}}+\cdots+\frac{p}{q^{\alpha+1}}\right) \\
& =\frac{1}{q^{\alpha+1}}\left\{1-\frac{q-p J_{1}}{q^{(m+1)\left(J_{1}+1\right)}}\left[1+\sum_{n=1}^{m}\left(-p q^{J_{1}}\right)^{n} \sum_{k=1}^{(m+1)\left(J_{1}+1\right)-n J_{1}-n} a_{n}(k)\right]\right\} \\
& +\frac{(\alpha+1) p\left(q-p J_{1}\right)}{q^{j_{u}+1-J_{1}}}+1-\frac{1}{q^{\alpha+1}}-\frac{q-p J_{1}}{q^{j_{u}+1}} \sum_{n=2}^{m+1}\left(-p q^{J_{1}}\right)^{n} \\
& \times\left[\sum_{k=1}^{j_{u}-n J_{1}-n+1} a_{n-1}(k)+\sum_{k=1}^{j_{u}-1-n J_{1}-n+1} a_{n-1}(k)+\cdots+\sum_{k=1}^{j_{u}-\alpha-n J_{1}-n+1} a_{n-1}(k)\right] \\
& =1-\frac{q-p J_{1}}{q^{j_{u}+1}}\left\{1+\sum_{n=1}^{m}\left(-p q^{J_{1}}\right)^{n} \sum_{k=1}^{(m+1)\left(J_{1}+1\right)-n J_{1}-n} a_{n}(k)-(\alpha+1) p q^{J_{1}}\right. \\
& +\sum_{n=2}^{m+1}\left(-p q^{J_{1}}\right)^{n}\left[a_{n}\left(j_{u}-n J_{1}-n+1\right)+a_{n}\left(j_{u}-1-n J_{1}-n+1\right)\right. \\
& \left.\left.+\cdots+a_{n}\left((m+1)\left(J_{1}+1\right)-n J_{1}-n+1\right)\right]\right\} \\
& =1-\frac{q-p J_{1}}{q^{j_{u}+1}}\left[1+\sum_{n=1}^{m+1}\left(-p q^{J_{1}}\right)^{n} \sum_{k=1}^{(m+1)\left(J_{1}+1\right)-n J_{1}-n} a_{n}(k)\right. \\
& \left.+\sum_{n=1}^{m+1}\left(-p q^{J_{1}}\right)^{n} \sum_{k=(m+1)\left(J_{1}+1\right)-n J_{1}-n+1}^{j_{u}-n J_{1}-n+1} a_{n}(k)\right] \\
& =1-\frac{q-p J_{1}}{q^{j_{u}+1}}\left[1+\sum_{n=1}^{\beta}\left(-p q^{J_{1}}\right)^{n} \sum_{k=1}^{j_{u}-n J_{1}-n+1} a_{n}(k)\right] \text {, }
\end{aligned}
$$


we know (15) holds for $\beta=m+1$. This completes the proof.

Remark. Comparing Theorem $3 \& 4$ one can see that the initial values (14) also satisfy (15) with $j_{u}=0$.

\subsection{Some discussions}

Having obtained the complete explicit expression of $\psi(u), u \geq 0$, one can calculate the ruin probabilities for given $u$ values as long as $N, K_{1}$ and $K_{2}$ are known. Before we present more numerical examples, we would like to make several remarks regarding the ruin probability calculation under our simplified NCD model.

\section{Remarks.}

3.1 We would like to continue our discussion in Remark 2.4 regarding the restrictions on parameters proposed in this paper. In the context of the simplified model, the assumptions are: $N-K_{1}$ being a multiple of $K_{2}$ and $\theta K_{1}=K_{2}$ being a positive integer. As mentioned in Remark 2.4, when the given problem does not satisfy these assumptions, we need to search for a set of values for $N, K_{1}$ and $K_{2}$ to get a close enough fit to the given real problem and then to convert the obtained probabilities to the ruin probability under consideration. Since normally $N$ is much larger than $K_{1}$ and $K_{2}$, we do have a certain level of freedom to choose appropriate $N, K_{1}$ and $K_{2}$ values. Without loss of generality, we would require the greatest common factor (GCF) of the chosen $N, K_{1}$ and $K_{2}$ to be 1 . Two simple examples of how to choose appropriate $N, K_{1}$ and $K_{2}$ values are:

(a) Given that the maximum total claim size in a year is 100, the full annual premium is 1 and we are considering a $10 \%$ discount $(\theta=0.9)$, then we let $N=1000, K_{1}=10$ and $K_{2}=9$ and the assumptions are satisfied. Denote $\xi(u)$ the ruin probability with initial surplus $u$ that is calculated based on these parameters. The original ruin probability can be obtained as $\psi(u)=\xi(10 u)$.

(b) For a $15 \%(\theta=0.85)$ discount, there are two options. The first one is $N=2009, K_{1}=20$ and $K_{2}=17$, and the claim size is 100.45 times the full premium level, where $\psi_{1}(u)=\xi_{1}(20 u)$. The second one is $N=1992, K_{1}=20$ and $K_{2}=17$, and the claim size is 99.6 times the full premium level, where $\psi_{2}(u)=\xi_{2}(20 u)$. Both cases are not exactly the same as the given situation but very close to it. One might choose either option, or simply use $\left(\psi_{2}(u), \psi_{1}(u)\right)$ as an interval covering the true $\psi(u)$ values. 
3.2 For some special discount levels, ie $\theta=1, \frac{1}{2}, \frac{1}{3}, \frac{1}{4}, \ldots$, the search for $N, K_{1}$ and $K_{2}$ values is trivial and will always lead to $K_{2}=1$. For instance, when $N=1000, \theta=\frac{1}{2}, K_{1}=10$, we can get $K_{2}=5$ and $N-K_{1}=$ $198 \times K_{2}$. A risk model proposed above with this set of parameters will have the same ruin probabilities as a model with $N=200, K_{1}=2$ and $K_{2}=1$. From the calculation point of view, these special discount levels can simplify all the previous results significantly. However, from the practical point of view, they mainly cover some very high levels of discount, i.e. $50 \%$, $66.7 \%, 75 \%$ and so on. In real life, general insurers may be happy to offer policyholders discounts on premiums up to $50 \%$ under certain terms and conditions (Lemaire and $\mathrm{Zi}[6]$ showed that there are many Bonus-Malus systems in European countries offering a $50 \%$ discount), but discounts over $50 \%$ are not often seen. Therefore, we are not going to put much attention on these cases in the sequel.

3.3 If the original problem proposes $N, K_{1}$ and $K_{2}$ values satisfying the two mentioned assumptions, but their $\mathrm{GCF}>1$, then we should proceed with smaller ones such that their $\mathrm{GFC}=1$. Then a conversion is needed to generate the original ruin probabilities.

3.4 We know for discrete risk models with non-integer irregular premiums/ surpluses, how to build a usable recursive framework for calculation purposes is still an open problem. The above method does not solve the general problem, but hopefully it could give readers a hint when searching for a better solution. As one can see from Theorem 4 that, the ruin probability $\psi(u)$ is independent of $i_{u}$ which means, for our risk model (1), if we use $K_{2}$ as a monetary unit, then the non-integer part out of the initial surplus $u$ will not have any impact on the corresponding ultimate ruin probability. Of course, this argument is based on the assumptions we made so far and it is not a general result.

\subsection{More numerical examples}

In the following, we shall examine the impact of the premium discounts on the ultimate ruin probability $\psi(u)$ through two numerical examples. We will illustrate how much the ruin probability will change with different level of discount on premiums under the assumptions of our model.

Example 2. In this example, we consider the following five cases:

(1) $N=4,000, K_{1}=40, K_{2}=33, p=0.008$; 
(2) $N=2,009, K_{1}=20, K_{2}=17, p=0.008$;

(3) $N=1,000, K_{1}=10, K_{2}=9, p=0.008$;

(4) $N=1,996, K_{1}=20, K_{2}=19, p=0.008$;

(5) $N=100, K_{1}=1, K_{2}=1, p=0.008$.

These cases share the same claim/premium ratio, i.e. $\frac{N}{K_{1}}$, that is 100 , where cases (2) and (4) are approximately equal to 100. The probabilities of having positive claims are also equal. Some quantities of interest regarding these five cases are summarised in Table 2. Note that case (5) is a simplified situation which leads to an ordinary compound binomial risk model with no premium discount.

\begin{tabular}{crcrrrr}
\hline Case & $N / K_{1}$ & $\theta$ & $E\left[X_{1}\right]$ & $E\left[C_{n}\right]$ & $\frac{E\left[C_{n}\right]}{E\left[X_{1}\right]}-1$ & $J_{1}$ \\
\hline$(1)$ & 100.00 & 0.825 & 32.000 & 33.056 & $3.30 \%$ & 120 \\
$(2)$ & 100.45 & 0.850 & 16.072 & 17.024 & $5.93 \%$ & 117 \\
$(3)$ & 100.00 & 0.900 & 8.000 & 9.008 & $12.60 \%$ & 110 \\
$(4)$ & 99.80 & 0.950 & 15.968 & 19.008 & $19.04 \%$ & 104 \\
$(5)$ & 100.00 & 1.000 & 0.800 & 1.000 & $25.00 \%$ & 99 \\
\hline
\end{tabular}

Table 2: Some characteristics of Example 2.

An obvious difference among these cases is about the safety loadings which are given in the column entitled $\frac{E\left[C_{n}\right]}{E\left[X_{1}\right]}-1$. Clearly the safety loading increases from case (1) to (5) significantly.

Before we go on to discuss the ruin probabilities, we need to clarify an important issue: the monetary scales (assumed to be measured by the full premium levels). To be able to compare the corresponding ruin probabilities among the cases on a fair basis, sets of equivalent initial surpluses should be used that are proportional to the corresponding full premium levels. For example, if we consider $u=1$ for case 5 , then we should consider $u=40$ for case $1, u=20$ for cases 2 and 4 and $u=10$ for case 3 . Having this issue clarified, Table 3 provides ruin probability $\psi(u)$ for selected $u$ values in each case given above, where the superscript $(i)$ corresponds to the case (i).

One can see from Table $2 \& 3$ that

- under the given conditions, the corresponding ultimate ruin probabilities $\psi(u)$ for the five cases are very different; 


\begin{tabular}{rccccc}
\hline$u$ & $\psi^{(1)}(40 u)$ & $\psi^{(2)}(20 u)$ & $\psi^{(3)}(10 u)$ & $\psi^{(4)}(20 u)$ & $\psi^{(5)}(u)$ \\
\hline 0 & 0.9677 & 0.9435 & 0.8871 & 0.8387 & 0.7984 \\
10 & 0.9645 & 0.9383 & 0.8767 & 0.8252 & 0.7815 \\
20 & 0.9609 & 0.9321 & 0.8653 & 0.8091 & 0.7633 \\
30 & 0.9569 & 0.9252 & 0.8528 & 0.7931 & 0.7435 \\
40 & 0.9526 & 0.9177 & 0.8392 & 0.7740 & 0.7220 \\
50 & 0.9478 & 0.9101 & 0.8244 & 0.7551 & 0.6987 \\
60 & 0.9425 & 0.9009 & 0.8082 & 0.7325 & 0.6735 \\
70 & 0.9367 & 0.8909 & 0.7904 & 0.7101 & 0.6462 \\
80 & 0.9303 & 0.8799 & 0.7711 & 0.6833 & 0.6167 \\
90 & 0.9226 & 0.8688 & 0.7479 & 0.6568 & 0.5846 \\
100 & 0.9150 & 0.8548 & 0.7255 & 0.6264 & 0.5515 \\
150 & 0.8876 & 0.8099 & 0.6510 & 0.5355 & 0.4513 \\
200 & 0.8586 & 0.7640 & 0.5771 & 0.4492 & 0.3616 \\
250 & 0.8313 & 0.7215 & 0.5140 & 0.3795 & 0.2913 \\
300 & 0.8044 & 0.6811 & 0.4565 & 0.3193 & 0.2344 \\
350 & 0.7784 & 0.6430 & 0.4063 & 0.2695 & 0.1885 \\
400 & 0.7536 & 0.6070 & 0.3608 & 0.2267 & 0.1517 \\
450 & 0.7293 & 0.5731 & 0.3211 & 0.1914 & 0.1221 \\
500 & 0.7060 & 0.5410 & 0.2852 & 0.1610 & 0.0982 \\
600 & 0.6611 & 0.4822 & 0.2255 & 0.1144 & 0.0636 \\
700 & 0.6194 & 0.4293 & 0.1782 & 0.0812 & 0.0412 \\
800 & 0.5802 & 0.3826 & 0.1409 & 0.0577 & 0.0266 \\
900 & 0.5436 & 0.3410 & 0.1114 & 0.0410 & 0.0172 \\
1000 & 0.5093 & 0.3039 & 0.0879 & 0.0291 & 0.0112 \\
2000 & 0.2648 & 0.0959 & 0.0084 & 0.0010 & 0.0001 \\
\hline
\end{tabular}

Table 3: Some values of $\psi(u)$ of Example 2. 
- case (5) has the lowest ruin probabilities \& the lowest (zero) discount, and case (1) has the highest ruin probabilities \& the highest discount level as well;

- the above point is consistent with the order of safety loadings of the five cases;

- as $u$ increases, the differences between the ruin probabilities of the five cases increase quickly to much higher than the differences between the discount levels.

Example 3. In this example, we reconsider the five cases given in Example 2 with changed probabilities for claims. One more case is added for the purpose of better comparisons. The parameters are given below:

(1) $N=4,000, K_{1}=40, K_{2}=33, p=0.00751$;

(2) $N=2,009, K_{1}=20, K_{2}=17, p=0.00770$;

(3) $N=1,000, K_{1}=10, K_{2}=9, p=0.00819$;

(4) $N=1,996, K_{1}=20, K_{2}=19, p=0.00866$;

(5) $N=100, K_{1}=1, K_{2}=1, p=0.00909$;

(6) $N=200, K_{1}=2, K_{2}=1, p=0.00457$.

In the first 5 cases, the full premiums and discount levels remain the same but claim size distributions vary across the five cases such that their safety loadings stay at the same level (10\%). Case (6) has a much higher discount level with the same safety loading as other cases. Table 4 summarises some quantities of interest regarding these six cases.

Similar to Table 3, Table 5 summarises $\psi(u)$ values of the above six cases for selected $u$ values. Comparing Table 3 and 5 one can see that the patterns shown in each table are totally different. In Table 5 , the ruin probabilities of case (1) - (5) stay very close to each other for the given $u$ values. Moreover, there is no fixed order among them either. Case (6) also shows similar ruin probability values which are consistently lower than other five cases on a marginal scale. It implies that under the assumptions given in Example 3, in particular the equal safety loading condition, ruin probabilities do not follow the same quantitative order as the premium discount levels. Also, the full premium in the first time period, which is not included in the safety loading calculation, seems having played a key role in reducing the ruin probabilities for case (6). 


\begin{tabular}{ccrrrr}
\hline Case & $\theta$ & $E\left[X_{1}\right]$ & $E\left[C_{n}\right]$ & $\frac{E\left[C_{n}\right]}{E\left[X_{1}\right]}-1$ & $J_{1}$ \\
\hline$(1)$ & 0.825 & 30.048 & 33.053 & $10 \%$ & 120 \\
$(2)$ & 0.850 & 15.476 & 17.023 & $10 \%$ & 117 \\
$(3)$ & 0.900 & 8.189 & 9.008 & $10 \%$ & 110 \\
$(4)$ & 0.950 & 17.281 & 19.009 & $10 \%$ & 104 \\
$(5)$ & 1.000 & 0.909 & 1.000 & $10 \%$ & 99 \\
$(6)$ & 0.500 & 0.913 & 1.005 & $10 \%$ & 198 \\
\hline
\end{tabular}

Table 4: Some characteristics of Example 3.

\begin{tabular}{rcccccc}
\hline$u$ & $\psi^{(1)}(40 u)$ & $\psi^{(2)}(20 u)$ & $\psi^{(3)}(10 u)$ & $\psi^{(4)}(20 u)$ & $\psi^{(5)}(u)$ & $\psi^{(6)}(u)$ \\
\hline 0 & 0.90826 & 0.90826 & 0.90826 & 0.90826 & 0.90826 & 0.90823 \\
10 & 0.89957 & 0.90012 & 0.89957 & 0.89992 & 0.89948 & 0.89944 \\
20 & 0.89006 & 0.89040 & 0.89006 & 0.88988 & 0.88987 & 0.88980 \\
30 & 0.87965 & 0.87975 & 0.87966 & 0.87987 & 0.87934 & 0.87924 \\
40 & 0.86825 & 0.86805 & 0.86826 & 0.86781 & 0.86780 & 0.86766 \\
50 & 0.85577 & 0.85634 & 0.85579 & 0.85580 & 0.85516 & 0.85498 \\
60 & 0.84211 & 0.84237 & 0.84214 & 0.84133 & 0.84131 & 0.84108 \\
70 & 0.82716 & 0.82704 & 0.82720 & 0.82692 & 0.82614 & 0.82584 \\
80 & 0.81079 & 0.81022 & 0.81084 & 0.80954 & 0.80951 & 0.80915 \\
90 & 0.79130 & 0.79337 & 0.79122 & 0.79224 & 0.79130 & 0.79086 \\
100 & 0.77223 & 0.77328 & 0.77221 & 0.77219 & 0.77218 & 0.77166 \\
150 & 0.70742 & 0.70801 & 0.70743 & 0.70744 & 0.70677 & 0.70596 \\
200 & 0.64203 & 0.64304 & 0.64200 & 0.64197 & 0.64195 & 0.64091 \\
250 & 0.58381 & 0.58521 & 0.58473 & 0.58367 & 0.58418 & 0.58294 \\
300 & 0.53142 & 0.53313 & 0.53139 & 0.53136 & 0.53135 & 0.52997 \\
350 & 0.48298 & 0.48492 & 0.48374 & 0.48287 & 0.48329 & 0.48180 \\
400 & 0.43967 & 0.44110 & 0.43965 & 0.43883 & 0.43961 & 0.43804 \\
450 & 0.39961 & 0.40121 & 0.39955 & 0.39951 & 0.39986 & 0.39824 \\
500 & 0.36320 & 0.36495 & 0.36374 & 0.36306 & 0.36371 & 0.36206 \\
600 & 0.30050 & 0.30243 & 0.30094 & 0.30038 & 0.30091 & 0.29926 \\
700 & 0.24862 & 0.25022 & 0.24899 & 0.24852 & 0.24896 & 0.24735 \\
800 & 0.20570 & 0.20702 & 0.20600 & 0.20524 & 0.20598 & 0.20445 \\
900 & 0.17019 & 0.17156 & 0.17015 & 0.16981 & 0.17042 & 0.16899 \\
1000 & 0.14058 & 0.14194 & 0.14077 & 0.14049 & 0.14100 & 0.13968 \\
2000 & 0.02106 & 0.02147 & 0.02112 & 0.02100 & 0.02119 & 0.02079 \\
\hline
\end{tabular}

Table 5: Some values of $\psi(u)$ of Example 3. 


\section{The deficit at ruin}

In this section we shall study the deficit at ruin in our given risk model (1) under the simplified NCD system considered in Section 3, mainly for simplicity reasons. Similar consideration could be taken in a more general situation, i.e. with more levels of premium discount, but with more tedious derivations involved. Note that Wagner [10] also considered this probability for the Markov risk model defined in [9].

If ruin occurs, then $\left|U_{T}\right|=y$ denotes the deficit at ruin satisfying $0<y \leq$ $N-K_{2}$. We define

$$
\varphi(u, y)= \begin{cases}\operatorname{Pr}\left(T<\infty,\left|U_{T}\right|=y \mid U(0)=u\right), & u \geq 0 \\ \delta_{-u, y}, & u<0\end{cases}
$$

which describes the probability that ruin occurs and the deficit at ruin equals $y$ where $\delta_{-u, y}$ is an indicator function of $\{-u=y\}$. Obviously, $\delta_{-u, y}=\delta_{u,-y}$. Further, $\varphi(u, y) / \psi(u)$ gives the distribution of the deficit at ruin given that ruin has occurred.

Under the same assumptions with respect to $N, K_{1}$ and $K_{2}$, i.e. the GCF of $N, K_{1}$ and $K_{2}$ is 1 and $N-K_{1}=J_{1} K_{2}$, we know that the potential deficit can only take values $y=1,2, \ldots, N-K_{2}$. Clearly, for $u \geq 0$,

$$
\psi(u)=\sum_{y=1}^{N-K_{2}} \varphi(u, y)
$$

Given $y>0$, considering the first time period we obtain the following recursion of $\varphi(u, y)$ :

$$
\varphi(u, y)=q \varphi\left(u+K_{2}, y\right)+p \varphi\left(u+K_{1}-N, y\right) .
$$

After assessing certain ranges of $u$ values, we have

$\varphi(u, y)= \begin{cases}\frac{1}{q} \varphi\left(u-K_{2}, y\right)-\frac{p}{q} \delta_{u-K_{2}+K_{1}-N,-y,} & K_{2} \leq u<K_{2}+N-K_{1}, \\ \frac{1}{q} \varphi\left(u-K_{2}, y\right)-\frac{p}{q} \varphi\left(u-K_{2}+K_{1}-N, y\right), & u \geq K_{2}+N-K_{1},\end{cases}$

where $\delta_{x, y}=1$ if $x=y$ and is 0 otherwise. The above recursive formula can be used to calculate $\varphi(u, y)$ if the initial values $\varphi(0, y), \ldots, \varphi\left(K_{2}-1, y\right)$ are known for any given $y$. Next we shall employ the same method as the one used in Section 3 to determine the initial values with the same assumptions regarding $N, K_{1}$ and $K_{2}$. 
Suppose that $n K_{2}>K_{2}+N-K_{1}$, then We have

$$
\begin{aligned}
& \varphi\left(n K_{2}, y\right)-\varphi(0, y)=\sum_{j=1}^{n}\left[\varphi\left(j K_{2}, y\right)-\varphi\left((j-1) K_{2}, y\right)\right] \\
& =p \sum_{j=1}^{J_{1}}\left[\varphi\left(j K_{2}, y\right)-\delta_{y,\left(J_{1}-j+1\right) K_{2}}\right]+p \sum_{j=J_{1}+1}^{n}\left[\varphi\left(j K_{2}, y\right)-\varphi\left(\left(j-1-J_{1}\right) K_{2}, y\right)\right] \\
& =p \sum_{j=1}^{n} \varphi\left(j K_{2}, y\right)-p \sum_{j=0}^{n-J_{1}-1} \varphi\left(j K_{2}, y\right)-p \sum_{j=1}^{J_{1}} \delta_{y,\left(J_{1}-j\right) K_{2}+K_{2}} \\
& =p \sum_{j=n-J_{1}}^{n} \varphi\left(j K_{2}, y\right)-p \varphi(0, y)-p \sum_{j=1}^{J_{1}} \delta_{y,\left(J_{1}-j\right) K_{2}+K_{2}}
\end{aligned}
$$

When $u \rightarrow \infty, \varphi(u, y)$ tends to zero, so letting $n \rightarrow \infty$ the above equation becomes

$$
\varphi(0, y)=p \varphi(0, y)+p \sum_{j=1}^{J_{1}} \delta_{y,\left(J_{1}-j+1\right) K_{2}} .
$$

Denote $i_{y}$ and $j_{y}$ the remainder and quotient from $y$ devided by $K_{2}$. One can see that

$$
\sum_{j=1}^{J_{1}} \delta_{y,\left(J_{1}-j+1\right) K_{2}}= \begin{cases}1 & \text { if } i_{y}=0,1 \leq j_{y} \leq J_{1} \\ 0 & \text { otherwise }\end{cases}
$$

Moreover, if $K_{1}<2 K_{2}$, i.e. $\theta>0.5$, then $N-K_{2}=N-K_{1}+K_{1}-K_{2}=$ $J_{1} K_{2}+K_{1}-K_{2}$, which leads to $j_{y} \leq J_{1}$. But if $\theta \leq 0.5$, then $j_{y} \leq J_{1}$ is not always satisfied. Therefore, we have the following result

$$
\varphi(0, y)=\left\{\begin{array}{cl}
\frac{p}{1-p} & \text { if } i_{y}=0,1 \leq j_{y} \leq J_{1} \\
0 & \text { otherwise }
\end{array}\right.
$$


Similarly, for $i=1,2, \ldots, K_{2}-1$,

$$
\begin{aligned}
& \varphi\left(n K_{2}+i, y\right)-\varphi(i, y)=\sum_{j=1}^{n}\left[\varphi\left(j K_{2}+i, y\right)-\varphi\left((j-1) K_{2}+i, y\right)\right] \\
= & p \sum_{j=1}^{J_{1}}\left[\varphi\left(j K_{2}+i, y\right)-\delta_{\left.y,\left(J_{1}-j\right) K_{2}+K_{2}-i\right]}\right. \\
& +p \sum_{j=J_{1}+1}^{n}\left[\varphi\left(j K_{2}+i, y\right)-\varphi\left(\left(j-1-J_{1}\right) K_{2}+i, y\right)\right] \\
= & p \sum_{j=1}^{n} \varphi\left(j K_{2}+i, y\right)-p \sum_{j=0}^{n-J_{1}-1} \varphi\left(j K_{2}+i, y\right)-p \sum_{j=1}^{J_{1}} \delta_{y,\left(J_{1}-j\right) K_{2}+K_{2}-i} \\
= & p \sum_{j=n-J_{1}}^{n} \varphi\left(j K_{2}+i, y\right)-p \varphi(i, y)-p \sum_{j=1}^{J_{1}} \delta_{y,\left(J_{1}-j\right) K_{2}+K_{2}-i} .
\end{aligned}
$$

When $u \rightarrow \infty, \varphi(u, y)$ tends to zero, so letting $n \rightarrow \infty$ the above equation becomes

$$
\varphi(i, y)=p \varphi(i, y)+p \sum_{j=1}^{J_{1}} \delta_{y,\left(J_{1}-j\right) K_{2}+K_{2}-i}
$$

Similarly, after considering all possible $i_{y}$ and $j_{y}$ values, we obtain, for $i=1,2, \ldots$, $K_{2}-1$,

$$
\varphi(i, y)=\left\{\begin{array}{cl}
\frac{p}{1-p} & \text { if } i_{y}=K_{2}-i, 0 \leq j_{y} \leq J_{1}-1 \\
0 & \text { otherwise }
\end{array}\right.
$$

In the following we shall derive explicit expressions of $\varphi(u, y)$ using previous results (17)-(19). Let $\gamma_{i_{u}, j_{u}, y} \hat{=} \varphi(u, y)$. The first case we consider is $i_{u}=i_{y}=0$. For $1 \leq j_{u}<J_{1}+1$, we have

$$
\begin{aligned}
& \gamma_{0, j_{u}, y}=\frac{1}{q} \gamma_{0, j_{u}-1, y}-\frac{p}{q} \delta_{\left(J_{1}-j_{u}+1\right) K_{2}, y} \\
& =\frac{1}{q}\left[\frac{1}{q} \gamma_{0, j_{u}-2, y}-\frac{p}{q} \delta_{\left(J_{1}-j_{u}+2\right) K_{2}, y}\right]-\frac{p}{q} \delta_{\left(J_{1}-j_{u}+1\right) K_{2}, y} \\
& \vdots \\
& =\frac{1}{q^{j_{u}}} \gamma_{0,0, y}-\sum_{k=1}^{j_{u}} \frac{p}{q^{k}} \delta_{\left(J_{1}-j_{u}+k\right) K_{2}, y} \\
& = \begin{cases}\frac{p}{q^{j u+1}} & 1 \leq j_{y} \leq J_{1}-j_{u}, \\
\frac{p}{q^{j u+1}}\left(1-p q^{J_{1}+1-j_{y}}\right) & J_{1}-j_{u}+1 \leq j_{y} \leq J_{1} \\
0 & \text { otherwise }\end{cases}
\end{aligned}
$$


For $J_{1}+1 \leq j_{u}<2\left(J_{1}+1\right)$ and $1 \leq j_{y} \leq J_{1}-j_{u}$, we have

$$
\begin{aligned}
\gamma_{0, j_{u}, y} & =\frac{1}{q} \gamma_{0, j_{u}-1, y}-\frac{p}{q} \gamma_{0, j_{u}-1-J_{1}, y} \\
& =\frac{1}{q} \gamma_{0, j_{u}-1, y}-\frac{p^{2}}{q^{j_{u}-J_{1}+1}} \\
& =\frac{1}{q^{2}} \gamma_{0, j_{u}-2, y}-\frac{2 p^{2}}{q^{j_{u}-J_{1}+1}} \\
& \vdots \\
& =\frac{1}{q^{j_{u}-J_{1}}} \gamma_{0, J_{1}, y}-\frac{\left(j_{u}-J_{1}\right) p^{2}}{q^{j_{u}-J_{1}+1}} \\
& =\frac{p}{q^{j_{u}+1}}-\frac{\left(j_{u}-J_{1}\right) p^{2}}{q^{j_{u}-J_{1}+1}}=\frac{p}{q^{j_{u}+1}}\left[1-\left(j_{u}-J_{1}\right) p q^{J_{1}}\right] .
\end{aligned}
$$

From (20) we can see that for $J_{1}+1 \leq j_{u}<2\left(J_{1}+1\right)$ and $J_{1}-j_{u}+1 \leq j_{y} \leq J_{1}$,

$$
\gamma_{0, j_{u}, y}=\frac{p}{q^{j_{u}+1}}\left(1-p q^{J_{1}+1-j_{y}}\right)\left[1-\left(j_{u}-J_{1}\right) p q^{J_{1}}\right]
$$

Put (21) and (22) together we have

$$
\gamma_{0, j_{u}, y}= \begin{cases}\frac{p}{q^{j}+1}\left[1-\left(j_{u}-J_{1}\right) p q^{J_{1}}\right] & 1 \leq j_{y} \leq J_{1}-j_{u} \\ \frac{p}{q^{j_{u}+1}}\left(1-p q^{J_{1}+1-j_{y}}\right)\left[1-\left(j_{u}-J_{1}\right) p q^{J_{1}}\right] & J_{1}-j_{u}+1 \leq j_{y} \leq J_{1} \\ 0 & \text { otherwise }\end{cases}
$$

Similar to Theorem 4, by induction, from (17), (20) and (23) one can prove that, for $i_{u}=i_{y}=0, j_{u} \geq 1$,

$$
\varphi(u, y)= \begin{cases}\frac{p}{q^{j_{u}+1}}\left[1+\sum_{n=1}^{\beta}\left(-p q^{J_{1}}\right)^{n} \sum_{k=1}^{j_{u}-n J_{1}-n+1} a_{n}(k)\right] & 1 \leq j_{y} \leq J_{1}-j_{u}, \\ \frac{p\left(1-p q^{J_{1}+1-j_{y}}\right)}{q^{j_{u}+1}}\left[1+\sum_{n=1}^{\beta}\left(-p q^{J_{1}}\right)^{n} \sum_{k=1}^{j_{u}-n J_{1}-n+1} a_{n}(k)\right] & J_{1}-j_{u}+1 \leq j_{y} \leq J_{1}, \\ 0 & \text { otherwise, }\end{cases}
$$

where $\beta$ and $a_{n}(k)$ have been defined in Theorem 4 .

The second case we shall consider is $1 \leq i_{u} \leq K_{2}-1$. In this case for $\varphi(u, y)$ to be nonzero, we need $i_{y}=K_{2}-i_{u}$. In the same manner as above, one can derive the following result of $\varphi(u, y)$ and the details of derivation will be omitted here: 
for $j_{u} \geq 1$

$\varphi(u, y)= \begin{cases}\frac{p}{q^{j u+1}}\left[1+\sum_{n=1}^{\beta}\left(-p q^{J_{1}}\right)^{n^{j_{u}-n J_{1}-n+1}} \sum_{k=1}^{p_{1}} a_{n}(k)\right] & 0 \leq j_{y} \leq J_{1}-j_{u}-1, \\ \frac{p\left(1-p q^{J_{1}-j_{y}}\right)}{q^{j_{u}+1}}\left[1+\sum_{n=1}^{\beta}\left(-p q^{J_{1}}\right)^{n_{j}-n J_{1}-n+1} \sum_{k=1}^{\beta} a_{n}(k)\right] & J_{1}-j_{u} \leq j_{y} \leq J_{1}-1, \\ 0 & \text { otherwise. }\end{cases}$

Thus, (18), (19), (24) and (25) form a complete set of explicit expressions of $\varphi(u, y)$ for $u \geq 0$ and $1 \leq y \leq N-K_{2}$.

\section{Conclusions}

This paper considered a discrete-time risk model with claim correlated premiums. At first, an NCD premium system with three levels of premium was considered, the full premium and discounted ones. Premiums in next time period depend on claim amounts in the previous period, the higher the claims the higher the premiums. Under this structure, a recursion approach was developed to calculating the ultimate ruin probabilities by introducing certain conditions on model parameters. A numerical example is given to illustrate the easiness of applying the recursive approach.

Thereafter, a simplified NCD system with only two levels of premium was examined in greater detail. Explicit results of the ruin probability were derived under similar parameter assumptions. Some discussions regarding a number of practical issues were made afterward with more numerical examples provided. These example have shown that:

- Given the claim distribution and full premium level, the higher the discount offered on premiums under the NCD mechanism, the higher the probability of ruin the insurer needs to face, because of the reduced safety loading level;

- When safety loadings are at the same level, the impact of premium discounts on ruin probabilities is marginal. The difference between the ruin probabilities with two significantly different premium discount levels could be largely devoted to the difference between initial premium amounts, which are excluded from the safety loading calculations. That is to say, the higher the initial premium compares with its discounted level, the more buffer the insurer gets from this initial premium for its own protection. 
To end this paper, the joint probability of ruin and the deficit at ruin was also studied in the simplified NCD case. Recursive formulae for the joint probabilities were derived and explicit results were obtained as well.

\section{Acknowledgments}

The authors are grateful to the anonymous referees whose constructive comments have led to substantial improvements of the article. The research of the second author was supported by a grant from National Natural Science Foundation of China (11426063) and a grant from Natural Science Foundation of Fujian Province (2015J05003). The research of the third author was supported by a grant from National Natural Science Foundation of China (11171164).

\section{References}

[1] L.B. Afonso, A.D. Reis, H.R. Waters, Numerical evaluation of continuoustime ruin probabilities for a portfolio with credibility updated premiums, ASTIN Bull. 40 (2010) 399-414.

[2] M. Denuit, X. Marchal, S. Pitrebois, J.F. Walhin, Actuarial modelling of claim counts: Risk classification, credibility and bonus-malus systems, John Wiley \& Sons Ltd., England, 2007.

[3] F. Dufresne, Distributions stationnaires d'un système bonus-malus et probabilité de ruine, ASTIN Bull. 18 (1988) 31-46.

[4] N.E. Frangos, S.D. Vrontos, Design of Optimal Bonus-Malus Systems with a Frequancy and a Severity Component On an Individual Basis in Automobile Insurance, ASTIN Bull. 31 (2001) 1-22.

[5] J. Lemaire, Bonus-malus systems in automobile insurance, Kluwer Academic Publishers, Boston/Dordrecht/London, 1995.

[6] J. Lemaire, H. Zi, A comparative analysis of 30 bonus-malus systems, ASTIN Bull. 24(2) (1994) 287-309.

[7] S. Li, D. Landriault, C. Lemieux, A risk model with varying premiums: Its risk management implications, Insur. Math. Econ. 60 (2015) 38-46.

[8] L. Tremblay, Using the Poisson Inverse Gaussian in Bonus-Malus Systems, ASTIN Bull. 22 (1992) 97-106. 
[9] C. Wagner, A note on ruin in two state Markov model, ASTIN Bull. 32 (2001) 349-358.

[10] C. Wagner, Time in the red in a two state Markov model, Insur. Math. Econ. 31 (2002) 365-372. 